\title{
Electrochemistry of fractal surfaces
}

Tamás Pajkossy

Research Centre for Natural Sciences, Hungarian Academy of Sciences

Magyar tudósok körútja 2, Budapest, Hungary, H-1117

e-mail: pajkossy.tamas@ttk.mta.hu

\begin{abstract}
The theories and expressions of electrochemistry have usually been derived by assuming some simple electrode geometry ensuring homogeneous current distribution along the surface. This is often not the case for practical electrodes: surface roughness, porosity and non-uniform surface activity cause current density inhomogeneities which in turn make the overall electrochemical behaviour complicated. One possible way of taking geometrical irregularities into account is to model the surfaces by fractals, whose most obvious feature is their blow-up symmetry. A number of theories exist for various electrochemical situations involving electrodes of irregular, fractal, geometries; two groups of are important as archetypes. The subject of the first is the frequency dependence of the impedance of a system comprised of a capacitive electrode of fractal geometry and a resistive electrolyte. The second is the time-dependence of the diffusional flux to an electrode of fractal geometry following an electrode potential jump making the electrode initially inactive to become reactive. The resulting equations, respectively for the frequency- and time-functions, are of power-law functions.
\end{abstract}

\section{Keywords:}

roughness, porousity, geometrical irregularity, fractal, Hausdorff dimension, current density distribution, inhomogeneity, diffusion, double layer capacitance, impedance, power-law distribution, constant phase element, capacitance dispersion

\section{Introduction}

Electrodes - just as other objects of Nature - are often of irregular geometry; which cannot be modelled by some simple shapes like a square or a sphere etc. The shape of these objects can often be approximated as fractals [1]. Fractals are geometrical objects whose most obvious feature is their blow-up symmetry: when zooming in, smaller and smaller features show up; and the details are similar to the whole: scale invariance or self-similarity is observed at least in some sense. Fractal geometry has provided an efficient tool to treat problems arising from irregular geometry, as shown by many examples in a number of branches of science, including two subjects of electrochemistry: (i) Electrodes of time-invariant, irregular geometry like ones with rough or partially active surfaces, porous bodies behave differently than those with uniform planar or spherical surfaces; (ii) Metal deposition and metal dissolution often changes surface geometry yielding branched-needles like dendritic structures rather than smooth layers, and creating caves, pits of irregular geometry, respectively [2]. These latter effects are much more complicated than the former ones, the published results are much more scattered hence these are beyond our present scope.

\section{Fractals}

Consider the classical concepts of geometry: the point, line segment, square and cube. Their $D_{\mathrm{E}}$ Euclidean dimension is $0,1,2$, and 3 , respectively. When they are magnified by a factor of $\beta$, their extent is magnified by $1, \beta^{1}, \beta^{2}$, and $\beta^{3}$, respectively. In general, the extent - named as Hausdorff measure, $M_{\mathrm{H}}$, of a classical object scales with $\beta^{D_{E}}$.

Consider an archetype-shape of fractal geometry, the von-Koch curve (Fig.1). This is a line of infinite length; and as of a line, its area is zero. Upon magnification by a factor of $\beta=1 / k$, (with $1 / 4<k<1 / 2)$ its length increases by a factor of 4 ; that is, by applying the above-mentioned scaling law of $M_{\mathrm{H}}, \beta^{d}=4$. Hence the exponent $d=\log (4) / \log (1 / k)$ is a non-integer number between 1 and 2 . 
In general, also for other objects of fractal geometry, the exponent $d$, might be in the full range of 0 to 3. Depending on the context, $d$ is called as Hausdorff, metric, similarity or fractal dimension; in what follows it will be denoted as $D_{\mathrm{f}}$.

For some $D_{\mathrm{E}}$ - dimensional classical shape, the extent (volume, area or length) can be determined by covering or filling the object with spheres of diameter $\varepsilon$; their number is $N(\varepsilon)$. The

$$
M=N(\varepsilon) * \varepsilon^{d}
$$

product, provided that $d$ has appropriately been chosen, for sufficiently small $\varepsilon$ has a finite, nonzero value. Then, $M_{\mathrm{H}}=M$. Were $d$ chosen other than $D_{\mathrm{E}}$ (e.g. we wanted to determine area by using length or volume standards that is one or three dimensional spheres) then infinite or zero $M$ value would be got.

Fractals are the objects for which Eq. 1 prevails with a $0<d<D_{\mathrm{E}}$ exponent (rather than $d=D_{\mathrm{E}}$ ). Carrying out the measurement procedure described above, analogously, $M_{\mathrm{H}}$ has finite positive value only if a certain, appropriate (usually non-integer) $d$ has been chosen; smaller or larger $d$ yields infinite and zero $M_{\mathrm{H}}$, respectively. The metric or Hausdorff dimension is defined by this measurement procedure and we can identify it as the exponent $d$ of Eq.1. As noted earlier, the metric or Hausdorff dimension of fractals is often called as fractal dimension, $D_{\mathrm{f}}$.

From the present point of view the very important consequence of the above definition of fractals is the following: Lets measure the surface of a fractal object of $1<D_{\mathrm{f}}<D_{\mathrm{E}}$ by covering it with $N$ spheres of $D_{\mathrm{E}}$ dimension of diameter $\varepsilon$. $\varepsilon$ represents the resolution of the measurement resolution, hence it is often called as "yardstick length". The area and the Hausdorff measure respectively are $A(\varepsilon)=N(\varepsilon) \varepsilon^{2}$ and $M_{\mathrm{H}}=N(\varepsilon) \varepsilon^{D_{\mathrm{f}}}$, hence the yardstick-length dependence of the measured area is

or

$$
A(\varepsilon)=M_{\mathrm{H}} \varepsilon^{2-D_{\mathrm{f}}}
$$

$$
\log [A(\varepsilon)]=\text { const }-\left(2-D_{\mathrm{f}}\right) \cdot \log (\varepsilon)
$$
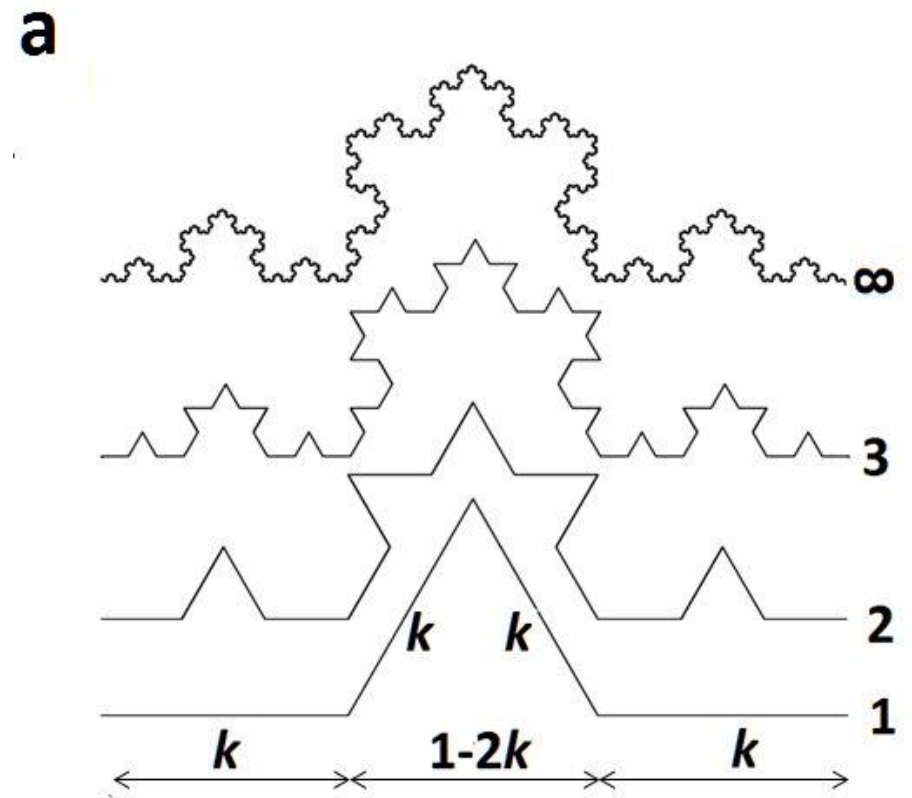

b

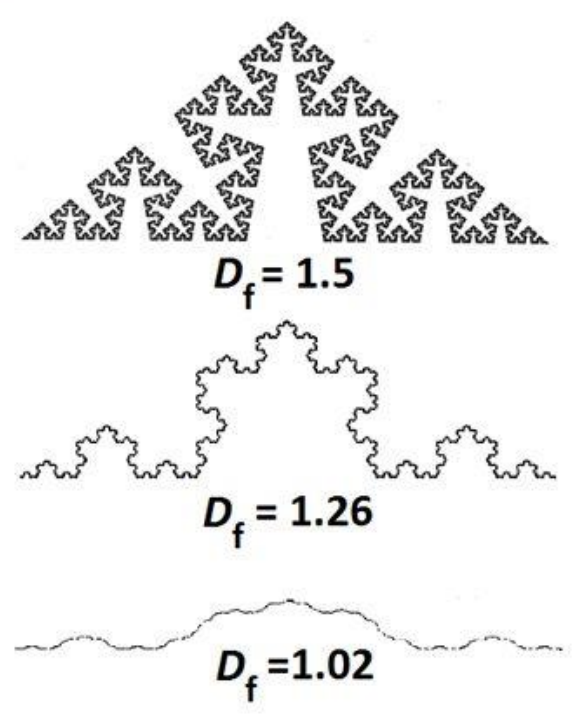

Fig.1: (a). Steps of construction of a von-Koch-curve. In the first step a line is divided to three parts of length $k, 1-2 k$, and $k$; the middle part is removed and two lines of lengths $k$ are drawn instead, in a triangular form. In the next and subsequent steps the procedure is repeated for the successively smaller line portions. Note that after $n$ steps the total length of the curve is $(4 \mathrm{k})^{n}$ times of that of the original; after infinite steps the total length is infinite. (b) Koch curves of varied $D_{\mathrm{f}}(k=0.257,0.333$, and 0.396 in increasing order of $D_{\mathrm{f}}$.) Note that apart their full sizes, no characteristic length of the structures can be identified - this is associated with that the size-distribution of the features of the curves is a power-law function. 
Many fractal structures and their properties are discussed in details in Ref. [1]. Here only a few aspects need to be mentioned: Fractality is in close connection with self-similarity or scaleinvariance, that is, details being similar to large parts show up upon magnification. This type of symmetry might appear in certain or in all directions, accordingly the fractal is self-affine or selfsimilar. The repetition of the similar units might follow a random or a regular pattern, as in Fig.1; in the former case we have a random fractal, for which the blow-up-symmetry prevails in an average sense. Finally, certain shapes might be fractal in a finite size range only, bordered by the so-called cut-offs.

Object, whose geometry can be readily approximated by fractals - at least in a certain size range are fairly frequent in Nature.

\section{Electrochemical processes on irregular surfaces}

There exist a number of phenomena in the area of electrode kinetics which are overcomplicated due to the effect that the points of the surface are not equally accessible for the participants of the processes. Even if the interfacial properties are the same at each surface points, the irregular electrode geometries often yield non-uniformity of the potential distributions and/or mass transport fluxes along the interfaces. These two effects can be understood through the analyses of simple, fundamental, related cases of (a) what is the impedance of an electrode in the complete absence of Faradaic reactions and (b) what is the time-dependence of a charge transfer reaction following an electrode potential jump. The answers are well-known for the cases of electrodes of planar or other simple geometries; however they are not simple if the electrode surface is rough, porous or irregular otherwise. These types of surfaces can be modelled by fractals.

\section{a. Impedance of a capacitive electrode of fractal geometry}

In general, if there is no Faradaic-reaction proceeding on an electrode, then the electric behaviour is governed jointly by that of the electrode/electrolyte interface and the electrolyte bulk. The former is capacitive due to the electrochemical double layer, the latter is resistive because of ions' conductance. Hence, in the simplest case, in a cell with parallelly placed planar electrodes, the current density is uniform along the interface; the parts of the electrodes are equally accessible to the current; the overall electrode impedance is a simple sum of the impedances of the capacitive interface and of the resistive electrolyte. This case is an exception rather than a rule. Usually, due to geometry reasons the electrode surfaces are not equally accessible for the current; the effective solution resistance differs along the electrode surface; the overall impedance will be a complicated complex function of the cell geometry parameters. This function can be analytically calculated for simple geometries like a disk embedded in an insulating plane. There exist cases when the electrode models' symmetry gives us a clue to predict that the cell impedance is a power-law function of frequency (a "constant phase element"). According to the theories this is the case when the electrode is uniformly porous (whose structure exhibits a translational symmetry) or fractal (i.e. has a blow-up symmetry). We note, however, that this constant phase element behaviour is usually completely masked by other effects of different, physico-chemical origin (which often exhibit also power-law frequency dependences [3]).

\section{b. Time dependence of the diffusion-limited current towards a fractal interface}

Consider a so-called Cottrell-experiment: an electrode is immersed in a homogeneous, unstirred solution: a highly conducting electrolyte containing some redox species. For the sake of simplicity let this species the oxidizable $\mathrm{R}$ of $c_{\mathrm{b}}$ bulk concetration. Initially the electrode is inert, no oxidation proceeds on it, hence no current flows. From the $t=0$ moment onward, the potential is set more positive at which $\mathrm{R}$ is oxidized with high rate, due to which a current starts to flow. The high oxidation rate causes that all oxidizable $\mathrm{R}$ molecules at the close vicinity of the electrode vanish (the surface concentration $c_{\mathrm{s}}=0$ ), and the concentration is lessened in a usually successively broadening zone called "depletion zone" or "diffusion layer". The current density at any point of the electrode is determined by the local diffusional flux of R, governed by Fick's first law, 
$\emptyset=-D \operatorname{grad}(c)$, where $D$ is the diffusion coefficient of the $\mathrm{R}$ species. The thickness of the depletion zone, $\delta$, can be defined through the concentration gradient $\operatorname{grad}(c)=\left(c_{b}-c_{b}\right) / \delta=$ $\left(-c_{b}\right) / \delta$.

To calculate analytically the time dependence of the current, $I(t)$, we have to solve the diffusion equation with the actual initial and boundary conditions. Such a calculation yields the temporal change of the concentration map; from this, $I(t)$ can be calculated. $I(t)$, in general, depends on the electrode geometry. In a simplest case, when the electrode is planar, and there are no convections are in the solution, $\delta=\sqrt{\pi t / D} ; I(t)$ is given by Cottrell's equation,

$$
I=-n F A D \operatorname{grad}(c)=n F A c_{b} \sqrt{D /(\pi t)}
$$

where $n \mathrm{~F}$ is the charge change when of one mole of $\mathrm{R}$ is oxidized, $A$ is the electrode area. The concentration map, i.e. equi-concentration surfaces are planes parallel to the electrode.

Lets consider now electrodes whose microscopic and macroscopic area are different. These are rough and partially active surfaces. For these cases the concentration maps are more complex than for the planar case. As it is illustrated in Figs. 2a and d, the area of the equi-concentration surfaces are equal to the microscopic and the macroscopic area, $\left(A_{\text {micr }}\right.$ and $\left.A_{\text {macr }}\right)$ respectively. According to this, as is illustrated in Figs $2 \mathrm{~b}$ and 2e, the time dependence of the current obeys Eq. 3 at short times and long times, however, one has to use $A_{\text {micr }}$ and $A_{\text {macr }}$ for $A$. In this sense, $A$ is the area of the surface referring to, say, to $c=c_{\mathrm{b}} / 2$ and thus $I$ is a measure of $A(t)$.

A transformation of the plots of Figs $2 b$ and $2 \mathrm{e}$ seems to be useful: The diffusion layer width, $\delta=\sqrt{\pi t / D}$ can be regarded as a yardstick length, $\varepsilon$, which in turn, determines the area of the equiconcentration surfaces. Thus, one can plot $A(\varepsilon) \approx I \sqrt{\pi t / D} /\left(n F A c_{b}\right)$ vs $\varepsilon \approx \sqrt{\pi t / D}$. Such transformed plots are seen in Figs. $2 \mathrm{c}$ and $2 \mathrm{f}$. Three time ranges can be separated. At short and long times the ordinate values are equal to the microscopic and macroscopic areas, repectively. In the intermediate time range, the area changes with yardstick length. Let us use the simple approximation that this dependence is linear. Note that a linear $\log [A(\varepsilon)]$ vs $\log (\varepsilon)$ corresponds to a fractal (cf.Eq.1b) and the fractal dimension can be readily calculated by using Eq.1b. In a similar vein, if we approximate the $\log (I)$ vs $\log (t)$ function in the intermediate time range by a straight line then it is equivalent to that we model the surface by some fractal. The slope of the $\log (I) \operatorname{vs} \log (t)$ function in the intermediate time range can be calculated by the following line of thoughts: The diffusional current is expressed by the Cottrell equation equipped with yardstick length dependent area. In logarithmic form:

$$
\log (I)=\log [A(\varepsilon)]+\log \left(n F A c_{b} \sqrt{D / \pi}\right)-1 / 2 \log (t)
$$

The area of a fractal surface depends on the yardstick length according to Eq.2. Since the yardstick length is $\varepsilon \approx \sqrt{\pi t / D}$ thus $\log (\varepsilon) \approx \log (\pi t / D) / 2$, Eq.4 combined with Eq 2 yields:

$$
\log (I)=\text { const }-\left(D_{\mathrm{f}}-1\right) / 2 \cdot \log (t)
$$

Eq. 5 expresses that in the intermediate time range the current is a power-law function of time, and the fractal dimension is included in the exponent, $\alpha=\left(D_{\mathrm{f}}-1\right) / 2$.

The following notes are due here:

1. Eq. 5 can be employed to the experimental determination of $D_{\mathrm{f}}$ of certain surfaces to be approximated by self-similar fractals. In the actual measurements, there is a number of other factors (effects of solution resistance, of double layer capacitance, slow charge transfer, spontaneous convections of the electrolyte) which may cause distorted $I(t)$ transients. However, the absence of the distortions can - and must - be proven by demonstrating that with the given experimental conditions on a flat electrode the $I(t)$ curve is very similar to that of Eq.3. [4].

2. The factor $\left(D_{\mathrm{f}}-1\right) / 2$ found in Cottrell-experiment results appears also in the expressions of other types of measurements with the same electrochemical system, e.g. in the expressions of cyclic voltammetry and Warburg impedance [5].

3. The transition section of the $\log (I)$ vs $\log (t)$ or the $\log (A)$ vs $\log (\varepsilon)$ curve, in principle, is not linear. The above approach, that is, replacing that section by a straight line is probably the simplest approximation. There exist much more exact theories for "realistic fractal 
electrodes" [6,7], leading to complicated equations with more-than-one parameters whose use might be difficult for analyzing the given electrochemical phenomena on rough electrodes.
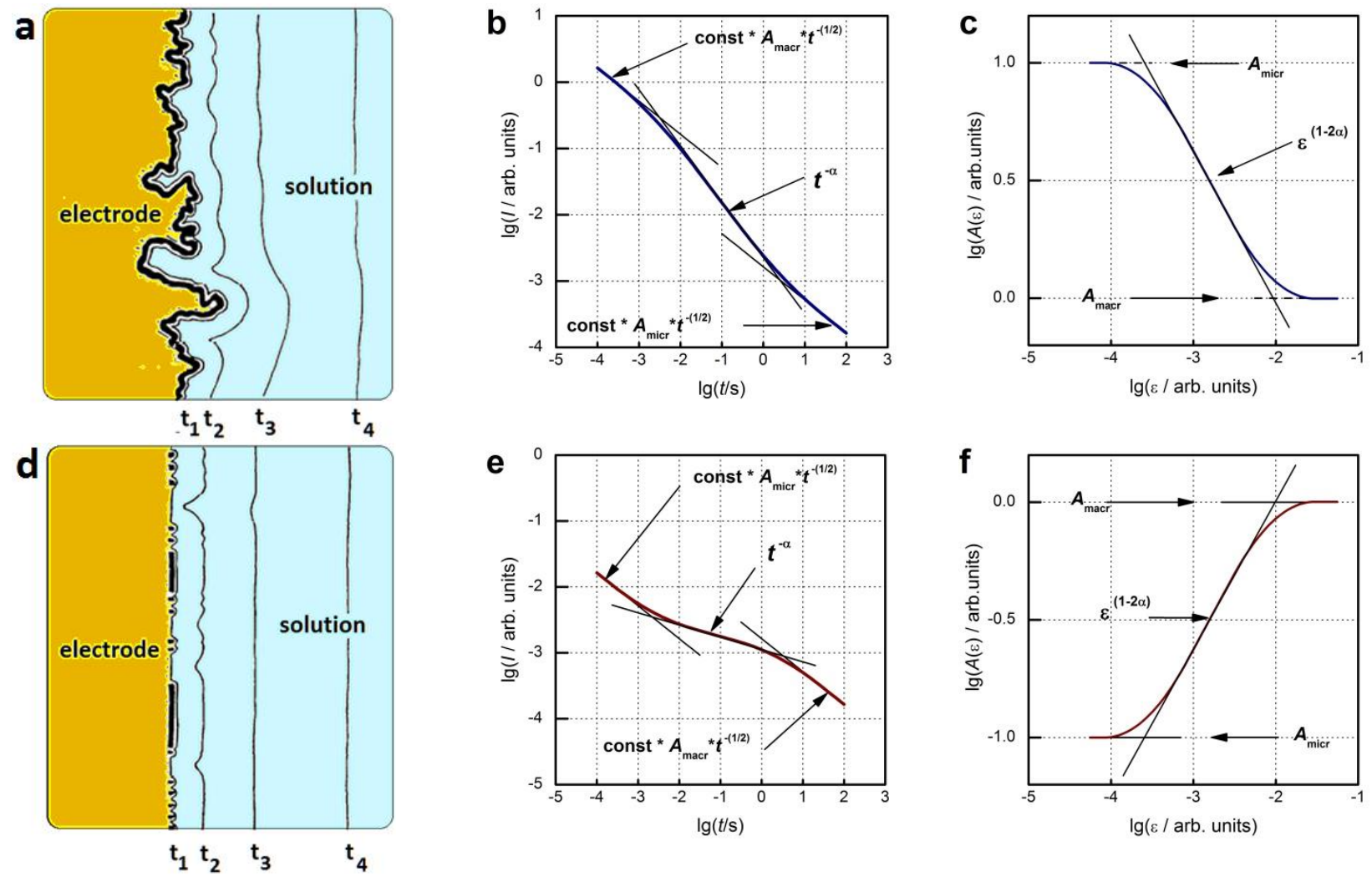

Fig.2. Concentration profiles (a) as a function of time in the vicinity of a rough electrode. (b) Diffusion controlled current as a function of time (c) The $A(\varepsilon)$ vs $\varepsilon$ plot. Figs (d), (e), and (f) are the same as (a) to (c), but for a partially active electrode.

\section{Concluding comments:}

1. Textbook expressions of the electrochemical phenomena have usually been derived assuming simple geometries, like planar interfaces. There exist a number of reasons why real electrodes exhibit properties different from those predicted by the ideal expressions irregular geometry is one possible reason. Real surfaces are seldom planar, just as seldom as ideal self-similar fractal surfaces. We stress that there is no one-to-one correspondence between power-law response functions (impedance and current transients) and fractal geometry.

2. The above, simple models simply demonstrate that for electrodes without characteristic sizes, the response functions exhibit no characteristic time or frequency.

\section{References:}

1 B.B. Mandelbrot: The Fractal Geometry of Nature, Freeman, San Francisco, 1982

2 J.-Y. Go, S.-I. Pyun, Fractal Approach to Rough Surfaces and Interfaces in Electrochemistry, in: Modern Aspects of Electrochemistry 39, C.G. Vayenas, R.E. White, (Eds), Springer Science \& Business Media, 2006. pp 167-229

3 T. Pajkossy: Impedance spectroscopy at interfaces of metals and aqueous solutions - surface roughness, CPE and related issues, Solid State Ionics 176 (25-28), 1997-2003 (2005) 
4 A. Imre, T. Pajkossy, and L. Nyikos: Electrochemical determination of the fractal dimension of fractured surfaces, Acta Metallurgica 40 (8), 1819-1826 (1992), doi: 10.1016/09567151(92)90168-E

5 T. Pajkossy, A.P. Borosy, A. Imre, S.A. Martemyanov, G. Nagy, R. Schiller, and L. Nyikos: Diffusion kinetics at fractal electrodes, J. Electroanal. Chem. 366, 69-73 (1994)

6 R. Kant, S.K. Rangarajan: Effect of surface roughness on diffusion-limited charge transfer, J. Electroanal. Chem. 368 (1994), 1, DOI: 10.1016/0022-0728(93)03069-2

7 S.K. Jha, A. Sangal, R. Kant, Diffusion-controlled potentiostatic current transients on realistic fractal electrodes, J. Electroanal. Chem., 615 (2008) 180-190, doi:10.1016/j.jelechem.2007.12.014 